Original Article

\title{
DEVELOPMENT AND VALIDATION OF RP-HPLC METHOD FOR THE DETERMINATION OF ALVIMOPAN IN RAT PLASMA
}

\author{
DEVI RAMESH ${ }^{1}$, MOHAMMAD HABIBUDDIN ${ }^{*}$
}

${ }^{1}$ Government Polytechnic College, Kancharapalem, Visakhapatnam, Andhra Pradesh, Pin530007 India, ${ }^{2}$ Shadan College of Pharmacy, Peerancheru, Hyderabad, Telangana, Pin 500086, India

Email: drhabib21@gmail.com

Received: 07 Aug 2018 Revised and Accepted: 07 Sep 2018

\begin{abstract}
Objective: The present investigation demonstrates a simple, sensitive and accurate high pressure liquid chromatographic (HPLC) method for the determination of alvimopan (AMP) in rat plasma.

Methods: The chromatographic separation was achieved within 10 min by using acetonitrile: potassium dihydrogen phosphate buffer $\mathrm{pH} 3.0$

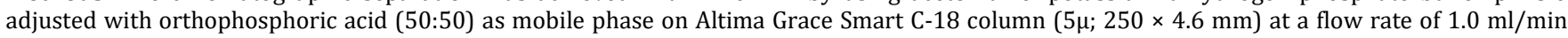
with injection volume $50 \mu \mathrm{l}$. The drug was extracted from plasma by liquid-liquid extraction using a mixture of methanol: acetonitrile (50:50) as a solvent. The retention times of drug and internal standard were found to be 5.17 and $6.74 \mathrm{~min}$, respectively. This method was validated as per the United States Food and Drug Administration (US-FDA) guidelines.
\end{abstract}

Results: The results of the validation parameters were found to be within the acceptance limits. The method was linear in the concentration range from $5-1000 \mathrm{ng} / \mathrm{ml}\left(\mathrm{r}^{2}=0.9998\right)$, and the extraction recovery was found to be $78.71 \pm 3.86 \%$ for AMP. The lower limit of quantification was found to be $5 \mathrm{ng} / \mathrm{ml}$, and the stability of recovered samples at different conditions was found to be more than $95 \%$.

Conclusion: The developed method possess good selectivity, specificity, there was no interference found in the plasma blanks at retention times of AMP and Internal Standard (IS). We found a good correlation between the peak area and concentration of the drug under prescribed conditions. Furthermore, the method can also be used to estimate the pharmacokinetic parameters of AMP.

Keywords: Alvimopan, Liquid-liquid extraction, Method development, Matrix effect, Plasma, Recovery, Stability, Validation

(C) 2018 The Authors. Published by Innovare Academic Sciences Pvt Ltd. This is an open-access article under the CC BY license (http://creativecommons.org/licenses/by/4.0/) DOI: http://dx.doi.org/10.22159/ijpps.2018v10i10.29001

\section{INTRODUCTION}

Narcotic medicines that are often used to reduce the pain caused by gastrointestinal surgery. However, these medicines can cause a variety of side effects, such as nausea, vomiting, constipation and stomach pain, which are further leads to delay recovery in patients undergoing gastrointestinal surgery. Therefore, medicines that are required to prevent the above-mentioned side effects without diminishing the pain-relieving effect of narcotic medicines. Alvimopan (AMP) (Entereg), the only drug approved by the Food and Drug Administration (FDA) for the treatment of postoperative ileus [1,2]. This drug behaves as a peripherally acting $\mu$-opioid antagonist. Since the AMP has limited ability to cross the blood-brain barrier, many of the undesirable side-effects of the narcotic medicines are minimized without affecting analgesia or precipitating withdrawal [3, 4]. In order to comprehend the antagonist effect of AMP further, it is imperative to determine the pharmacokinetic parameters through estimation of the AMP in plasma using simple estimation methods.

Several liquid chromatography-tandem mass spectrometric (LCMS/MS) assay methods have been employed for the determination of various drugs in the form of single and combined dosage forms [6-9]. In addition, estimation of AMP in plasma and pharmaceutical formulation has also been carried out using LC-MS/MS method. In general, this method is highly sensitive to separate and identify a multitude of compounds in low concentration in a complex mixture with little assay optimization $[10,11]$. However, this method has a variety disadvantages. Specifically, it requires an experienced technician, not portable, expensive and has only moderate throughput. Therefore, it is important to develop a method, which is simple and inexpensive for the estimation of AMP in plasma for routine analysis.

The present contribution provides a simple and regular estimation method for determination of AMP in rat plasma using high-pressure liquid chromatography (HPLC). [fig. 1] gives the molecular structure of AMP. As the above-mentioned, AMP is the only narcotic antagonist approved by the FDA for the treatment of postoperative surgery of gastro intestine. In order to estimate the pharmacokinetic parameters, we made an attempt to determine the AMP in the rat plasma using routine HPLC method.

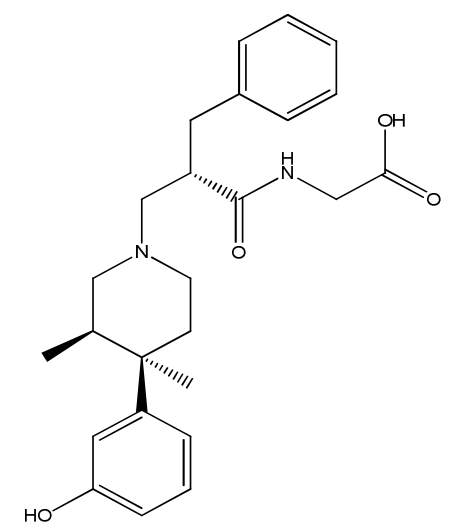

Fig. 1: Chemical structure of 2-([(2S)-2-([(3R, 4R)-4-(3hydroxyphenyl)-3,4-dimethylpiperidin-1-yl]methyl)-3phenylpropanoyl]amino)acetic acid

\section{MATERIALS AND METHODS}

Chemicals and standards

Alvimopan (AMP) procured from Aurobindo Pharma (Hyderabad, India), aceclofenac (ACF) purchased from S. L. drugs (Hyderabad, 
India). Purified water is prepared using a Millipore direct- $Q 3$ water purification system. Acetonitrile and methanol of HPLC grade, potassium dihydrogen phosphate, and orthophosphoric acid were purchased from Merck Ltd. (Mumbai, India).

\section{Preparation of standard solutions}

Preparation of AMP standard stock solution: $50 \mathrm{mg}$ of AMP was weighed accurately and dissolved in $50 \mathrm{ml}$ volumetric flask and made up to mark with methanol. The stock solution was diluted with the mobile phase solution when required.

Preparation of Internal standard stock solution: $10 \mathrm{mg}$ of ACF was weighed accurately dissolved in $10 \mathrm{ml}$ volumetric flask and made up to mark with methanol.

Preparation of phosphate buffer: Accurately weighed $2.72 \mathrm{~g}$ of potassium dihydrogen orthophosphate dissolved in $1000 \mathrm{ml}$ of HPLC grade water and $\mathrm{pH}$ adjusted to 3.0 with orthophosphoric acid and sonicated.

\section{Sample preparation}

A $0.25 \mathrm{ml}$ aliquot of plasma sample was spiked with $25 \mu \mathrm{l}$ of drug (AMP) and $25 \mu \mathrm{l}$ of IS, vertexes for $5 \mathrm{~min}$. Added the $2 \mathrm{ml}$ of a mixture of methanol: acetonitrile (50:50), vertexed for $5 \mathrm{~min}$ and the mixture was centrifuged for $15 \mathrm{~min}$ at $5000 \mathrm{rpm}$ at $20^{\circ} \mathrm{C}$. The supernatant liquid was separated and evaporated under nitrogen gas at $45^{\circ} \mathrm{C}$. It reconstituted the residue with $0.5 \mathrm{ml}$ of mobile phase and vertexed. The sample was filtered through $0.45 \mu$ syringe filter, then, loaded the sample into autoinjector vial and $50 \mu \mathrm{l}$ of the sample injected onto HPLC system.

\section{Method validation}

The validation of the developed method was carried out as per US FDA guidelines for selectivity, linearity, sensitivity, accuracy, precision, recovery and stability $[12,13]$.

\section{Selectivity}

The selectivity was studied by comparing the chromatograms of six different batches of plasma sample obtained from six independent lots of control plasma along with six extracted LOQ-QC samples. The method is selective if there is no interfering peak present at the retention time of the drug or IS.

\section{Linearity}

A calibration curve is the relationship between instrument response and known concentrations of the drug. The series of standards were prepared by spiking the required volume of working standard to $0.25 \mathrm{ml}$ of plasma to yields the concentrations of 5, 20, 50,100, 200, 400, 500 and $1000 \mathrm{ng} / \mathrm{ml}$. Extracted the drug from plasma and injected the each sample into HPLC. The linearity graph was plotted between the peak area ratios (y-axis) of AMP to IS versus the known concentration (x-axis) of AMP in plasma.

\section{Limit of quantification}

The lower limit of quantification (LLOQ) is the lowest concentration giving a signal-to-noise ratio of at least 10 -folds, with an accuracy of $80-120 \%$ and precision of $20 \%$ to its nominal value. This is determined by analyzing 10 times of LLOQ concentration and calculated the accuracy and precision.

\section{Accuracy and precision}

Intra-and inter-day accuracy and precision for this method was determined at three different concentration levels on three different days. The accuracy and precision were expressed as percentage accuracy and coefficient of variation (\% CV) respectively. The accuracy was calculated as follows.

$$
\text { Accuracy }(\%)-\frac{\text { concentration found }}{\text { Nominal concentration }} \times 100
$$

The coefficient of variation, \% CV was calculated as follows

$$
\% \mathrm{CV}=\frac{\text { Standard deviation }}{\text { Mean }} \times 100
$$

The accuracy determined at each concentration level must be within in $15 \%$ and the precision around the mean value must not exceed $15 \%$ except the LLOQ where it must be within $20 \%$ of the $\% \mathrm{CV}$.

\section{Recovery and matrix effect}

Recovery is the detector response obtained from an amount of the analyte added to and extracted from the biological matrix, compared to the detector response obtained for the true concentration of the standard. It is accessed by comparing the mean peak areas of extracted $\mathrm{LQC}, \mathrm{MQC}$ and $\mathrm{HQC}$ samples to the one obtained after the direct injection of a solution with corresponding concentration. The recovery of the drug was calculated by using the following formula:

$$
\text { Recovery }(\%)=\frac{C}{B} \times 100
$$

Matrix effect (ME) can be expressed as the suppression or enhancement of ionization of analyte by the presence of matrix components in the biological samples; quantitatively it can be termed as matrix factor. The matrix effect was calculated by using the following formula:

$$
\text { Matrix Effect }(\%)=\frac{R}{A} \times 100
$$

In this study, the peak area of AMP obtained by direct injection of standard solution as A, the corresponding standard solution of AMP spiked after extraction into plasma, injected into HPLC, the peak area of AMP as B, standard solutions spiked in plasma before extraction and followed extraction procedure and injected into HPLC, the peak area of AMP as $C$. The matrix effect and extraction recovery of the IS and AMP determined according to Matuszewski, B. K, et al. [14].

\section{Hemolytic effect}

The hemolysis effect was investigated according to the procedure described by Nicola C Hughes et al. [15]. The LQC and HQC of analyte were spiked with plasma, and hemolysed plasma samples were extracted and analyzed. If there is less than 15\% difference of analyte found in the plasma as compared to hemolysed plasma, indicates no hemolytic effect [15].

\section{Stability}

The stability of the drug solution was determined for short-term by keeping at room temperature $\left(25^{\circ} \mathrm{C}\right)$ for $24 \mathrm{~h}$. Autosampler stability was determined by storing the samples for $22 \mathrm{~h}$ in the autosampler. FreezeThaw stability: The plasma sample spiked with drug and kept in freeze ($\left.20^{\circ} \mathrm{C}\right)$ for $24 \mathrm{~h}$ and thawed $\left(25^{\circ} \mathrm{C}\right)$ for $24 \mathrm{~h}$. The same procedure repeated for two more cycles then followed the extraction procedure and analyzed. Wet extract samples were processed, reconstituted and kept on the bench at room temperature and analyzed after $24 \mathrm{~h}$ for stability. Dry extract samples were processed, after evaporation, which kept on the bench at room temperature and analyzed after $24 \mathrm{~h}$ to check their stability. Each sample injected into HPLC and concentrations obtained were compared with the nominal values of the $\mathrm{QC}$ samples.

\section{RESULTS AND DISCUSSION}

\section{Method development \\ Method optimization}

The chromatographic method was optimized as a mixture of $20 \mathrm{mmol}$ potassium dihydrogen phosphate buffer $(\mathrm{pH} 3.0)$ and acetonitrile $50: 50$ $\mathrm{v} / \mathrm{v}$ ) at a flow rate of $1 \mathrm{ml} / \mathrm{min}$ with detection wavelength of $261 \mathrm{~nm}$ by using Altima Grace Smart C-18 column by changing various parameters on trial and error basis. During the method optimization, water and phosphate buffer in various strengths are tried along with methanol and acetonitrile as organic solvent. The mobile phase composition of 50:50 $\mathrm{v} / \mathrm{v}$ acetonitrile: buffer was given good resolution, retention times of AMP and IS with a minimal tailing factor in acceptable range. The method was optimized with the mobile phase composition of acetonitrile and phosphate buffer 50:50 (v/v). The effect of buffer strength on the determination of drug was studied by different buffer strengths $(10,20$ and $50 \mathrm{mmol})$. There were no significant changes in the chromatographic response and peak shape with a change in buffer molarity. A buffer molarity of $20 \mathrm{mmol}$ was selected for further analysis. 
After several trials, the method was optimized as a mixture of 20 mmol potassium dihydrogen phosphate buffer $(\mathrm{pH} 3.0)$ and acetonitrile $(50: 50 \mathrm{v} / \mathrm{v})$, at a flow rate of $1 \mathrm{ml} / \mathrm{min}$, at $261 \mathrm{~nm}$ for run time $15 \mathrm{~min}$. These chromatographic conditions achieved a satisfactory resolution, retention time and tailing for AMP. The [fig. 2] shows that standard chromatogram of AMP along with the internal standard (IS).

Two extraction methods were tried for sample preparation i.e. protein precipitation (PPT), liquid-liquid extraction (LLE). These methods were studied for their effect on matrix sensitivity and resolution. PPT was the least effective sample preparation technique, often resulting in significant matrix effects due to the presence of many residual matrix components. LLE provided clean extract and reproducible recovery of AMP and IS. So, liquid-liquid extraction was employed in this assay development. Several organic solvents like ethyl acetate, dichloromethane, acetonitrile, methanol, and their mixtures were tried for extraction. Finally, the mixture of methanol and acetonitrile (50:50) was found to be suitable and produced a clean chromatogram for blank plasma samples with the best recovery of ARM, also possesses least matrix effect and cost-effective. It also quickly evaporate consumes less time for extraction.
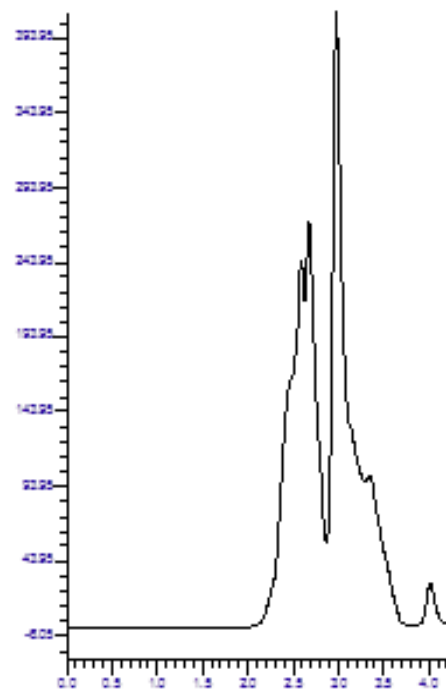

Blank Plasma
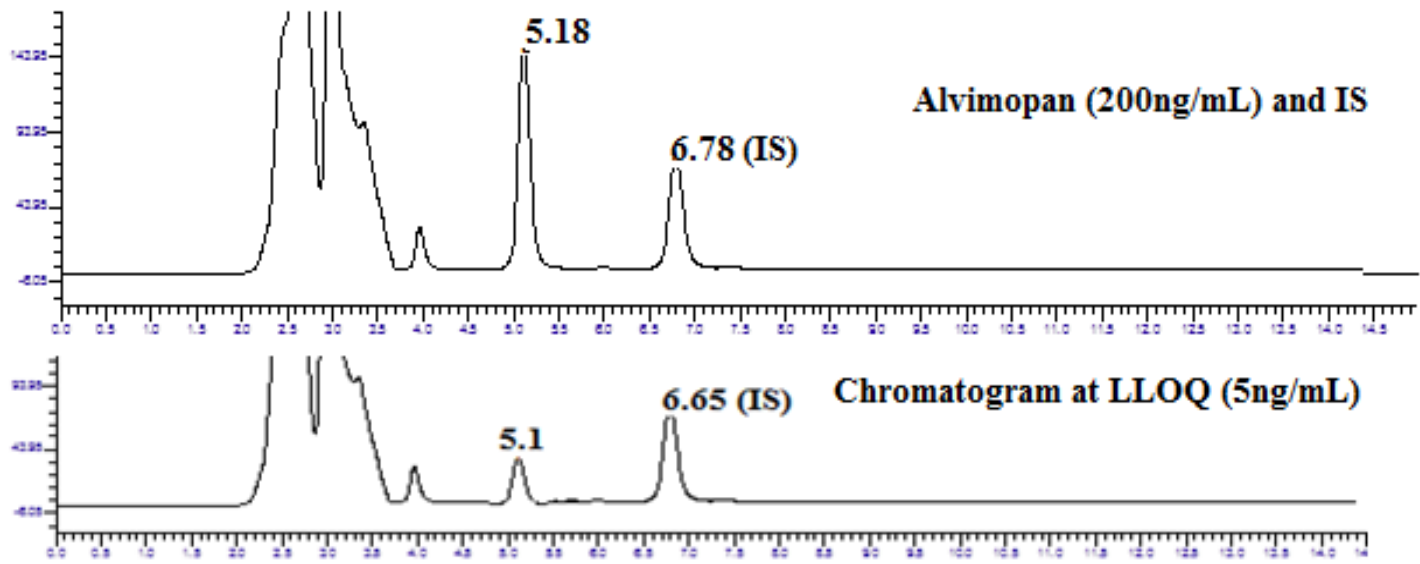

Fig. 2: The chromatograms of blank plasma and plasma spiked with drug (AMP) and IS

We investigated several compounds to find a suitable IS, by preparing standard reference solution containing alvimopan along with aceclofenac (ACF) and telmisartan (TEL), (which were easily available for us) in the above selected mobile phase. It was injected six times on to HPLC and observed the peak shape, response, and interference of these peaks with the analyte. TEL as internal standard produced good response but poor consistent results obtained with longer retention time. In another evaluation of ACF as internal standard produced sharp peak, no interference with analytes peak as well as reproducible results was obtained. So, ACF was employed in this study as an internal standard. Hence, ACF was selected as internal standard for this study.

\section{System suitability}

The system suitability of the method was done by working stock standard of individual drugs (AMP and IS) were injected HPLC to determine the individual retention times of drugs. Then working standard solution was injected five times and we considered relative standard deviation (RSD) for five consecutive injections $\leq 2$, the resolution between two adjacent peaks $\geq 2$ and tailing factor $<2$ acceptable values [16]. Resolution (R), relative standard deviation from five replicate injections of working standard mixture solution, tailing factor (T) and retention time drug was presented in [table 1]. System suitability test confirmed that the chromatographic system was adequate for the analysis planned to be done. Then, the method was validated for various validation parameters according to the US FDA guidelines $[12,13]$.

\section{Method validation}

\section{Selectivity and specificity}

The developed method was found selective for both AMP and IS, as no interference was detected at the respective retention times. The chromatogram of blank extracted from plasma and chromatogram of plasma spiked with AMP and IS at LLOQ and $200 \mathrm{ng} / \mathrm{ml}$ are shown in [fig. 2]. The specificity of the present method was established by 
checking the interference of AMP retention time with that of IS. This was done by injecting six replicates of matrix blank with IS. The interference of IS retention time caused by AMP, this was done by injecting 6 replicates of medium concentration of AMP. In this study, there was no peak interference of AMP or IS retention time [fig. 2]. This clearly shows the specificity and selectivity of the method.

\section{Carryover effect}

The carryover effect of the present method was established by using six injections of plasma blank and an upper limit of quantification (ULOQ) of AMP. These samples were analyzed alternately to check any carryover in the blank sample. In this study, there were no such effects observed.

Table 1: System suitability parameters of AMP

\begin{tabular}{lll}
\hline Parameters & AMP & IS \\
\hline Retention time (min) & $5.17 \pm 0.04$ & $6.74 \pm 0.04$ \\
Tailing factor & $1.13 \pm 0.01$ & $1.20 \pm 0.01$ \\
Theoretical plates & $5881 \pm 101$ & $7414 \pm 131$ \\
Peak area & $49891.1 \pm 375.8$ & $45431 \pm 310$ \\
\hline
\end{tabular}

Note: Values are expressed in mean \pm SD $(n=6)$

\section{Matrix effect}

The matrix effect was studied at three concentration levels (LQC, $\mathrm{MQC}$, and $\mathrm{HQC}$ ), there was no significance difference in peak area of the drug in the presence and absence of matrix ions. The matrix factor and matrix effect were found to be 0.99 and $>96 \%$ [table 2]. The results of ME also found within the acceptance limits indicates there was no significant matrix effect for AMP found in this method.

Table 2: Matrix effect (ME) on the extraction of AMP from plasma

\begin{tabular}{|c|c|c|c|}
\hline Standard(ng/ml) & MF (Matrix factor) & \% RSD & $\% \mathrm{ME}$ \\
\hline LQC(15) & $96.35 \pm 1.96$ & 2.04 & 0.96 \\
\hline MQC (250) & $97.88 \pm 0.99$ & 1.01 & 0.98 \\
\hline HQC $(750)$ & $102.77 \pm 3.79$ & 3.69 & 1.03 \\
\hline
\end{tabular}

Note: Values are expressed in mean \pm SD, Number of the sample $(n=3)$

\section{Recovery}

The extraction recovery was determined at three concentration levels (LQC, MQC, and HQC) for AMP and IS by comparing the peak area of AMP obtained by injecting the standard drug spiked with plasma followed extraction, the peak area of AMP obtained by injection standard drug of same concentration. The extraction recoveries were found to be $78.71 \pm 3.86 \%$ and $68.60 \pm 0.62 \%$ for AMP and IS respectively. The data represented in [table 3].

The hemolysis effect was studied by spiking the LQC and HQC with hemolysed blood. The hemolysed QC samples were extracted and analyzed. We could not find any hemolysis effect in this method.

Table 3: Extraction recovery of AMP and IS from rat plasma

\begin{tabular}{|c|c|c|c|c|}
\hline Drug & Standard (ng/ml) & Extracted matrix standard average peak area & Standard drug average Peak area & \% Recovery \\
\hline \multirow{3}{*}{ AMP } & LQC (15) & $6924.33 \pm 54.3$ & $8441 \pm 111$ & 82.03 \\
\hline & MQC (250) & $59061 \pm 1118.06$ & $74177 \pm 1557.32$ & 79.62 \\
\hline & HQC (750) & $176561 \pm 998.43$ & $237092 \pm 10444$ & 74.47 \\
\hline \multicolumn{4}{|c|}{ Average recovery } & $78.71 \pm 3.86$ \\
\hline \multirow[t]{3}{*}{ ACF (IS) } & LQC (15) & $45032.33 \pm 735.41$ & $65674.7 \pm 391.79$ & 68.56 \\
\hline & MQC (250) & $45115.3 \pm 612.33$ & $65157.7 \pm 259.43$ & 69.24 \\
\hline & HQC (750) & $44432 \pm 241.16$ & $65343.7 \pm 579.23$ & 67.99 \\
\hline \multicolumn{3}{|c|}{ Average Recovery } & & $68.60 \pm 0.62$ \\
\hline
\end{tabular}

Values are expressed in mean $\pm \mathrm{SD}, \mathrm{n}=6$

\section{Linearity}

The linearity of this method was evaluated by linear regression analysis, using the least square method. The peak area ratio of the drug and internal standard was used for the quantification of AMP. Calibration curves were linear in the concentration range of 5-1000 $\mathrm{ng} / \mathrm{ml}$ with a correlation coefficient $\left(\mathrm{r}^{2}\right)$ of 0.999 and the mean regression equation was: $y=0.005 x+0.082$, Where $y$ is the peak ratio and $\mathrm{x}$ is the plasma concentration of AMP. The linearity graph was shown in [fig. 3]. The linearity range of present method (5-1000 $\mathrm{ng} / \mathrm{ml}$ ) was useful for the determination of AMP in rat plasma.

\section{Sensitivity}

The standard chromatogram of AMP at LLOQ level was presented in [fig. 2]. The lower limit of quantitation (LLOQ) was found to be 5 $\mathrm{ng} / \mathrm{ml}$. The percent accuracy of LLOQ was $94.60 \pm 7.57 \%$ and precision denoted by $\%$ RSD was $8.00 \%$.

\section{Intra-day and inter-day precision and accuracy}

The intra-and inter-day precision and accuracy of this assay were determined by analyzing replicates of QC samples at three concentrations on 6 different days. The coefficients of variation for the intra-and inter-day precision were $<3.03 \%$. The intra-and inter-day accuracies were $98.28-102.99 \%$. The low levels of coefficients of variation i.e.: $1.86 \%-3.03 \%$ [table 4 ] indicate the method is accurate and precise.

\section{Robustness}

Robustness of the method was done by changing slight variation in the parameters like mobile phase composition, flow rate, and wavelength. Present method didn't show any significant change when the critical parameters were modified. The tailing factor of the drug was always less than 2.0 and the components were well separated under all the changes carried out. Considering the 
modifications in the system suitability parameters and the specificity of the method, as well as carrying the experiment at room temperature may conclude that the method conditions were robust.

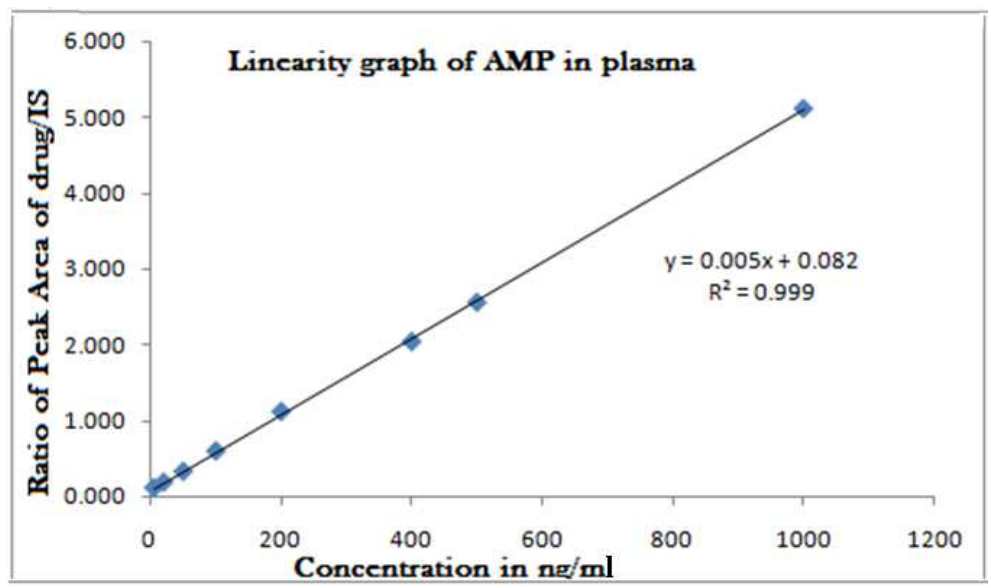

Fig. 3: Linearity graph of Alvimopan, $n=6$

Table 4: Intra-day and inter-day accuracy and precision of AMP in plasma

\begin{tabular}{llll}
\hline & Standard (ng/ml) & Average practical concentration & Accuracy mean \pm SD \\
\hline Intra-day & LQC (15) & $15.45 \pm 0.38$ & $102.99 \pm 2.56$ \\
$(\mathrm{n}=6)$ & MQC (250) & $245.71 \pm 4.56$ & $98.28 \pm 1.82$ \\
& HQC (750) & $752.94 \pm 22.80$ & $100.39 \pm 3.04$ \\
Inter-day & LQC (15) & $14.95 \pm 0.41$ & $99.64 \pm 2.74$ \\
$(\mathrm{n}=9)$ & MQC (250) & $248.84 \pm 5.53$ & $99.54 \pm 2.21$ \\
& HQC (750) & $753.03 \pm 21.46$ & 1.86 \\
\hline
\end{tabular}

Note: Values are expressed in mean $\pm S D$

\section{Ruggedness}

Ruggedness was studied along with precision and accuracy of batches where the effect of column change and analyst change were observed. The observed value for column variation and results obtained for precision and accuracy were within the acceptance criteria (i.e. there were no significance changes in the retention time, recovery and precision of the drug).

\section{Stability studies}

The stability of drug was studied at different conditions for quality control $(\mathrm{QC})$ of samples. The samples were analyzed and compared with freshly analyzed QC samples, no difference was found in accuracy and precision. There were no documented reports in the literature about the stability of AMP in plasma. To find any changes in stability of AMP in plasma, we carried out stability studies at different conditions like freeze-thaw, wet extract, dry extract stability etc. In the present method we studied the stability of AMP in plasma for $24 \mathrm{~h}$, freeze-thaw stability after three cycles and other stability studies. These studies enlighten the information regarding degradation of the drug during the analysis and storage of plasma samples. From these results stability of samples represented [table 5], the accuracy of all samples stability was found to be $>95 \%$ indicating that there was no degradation of the drug at different conditions.

Table 5: Data of different stability studies of AMP in plasma

\begin{tabular}{|c|c|c|c|c|}
\hline Stability & Standard (ng/ml) & Average practical concentration & Accuracy & \% RSD \\
\hline \multirow{3}{*}{ Freeze and thaw stability } & LQC (15) & $14.67 \pm 0.33$ & $97.81 \pm 2.24$ & 2.29 \\
\hline & MQC (250) & $251.05 \pm 0.38$ & $100.52 \pm 0.15$ & 0.15 \\
\hline & HQC (750) & $747.74 \pm 27.06$ & $99.70 \pm 3.61$ & 3.62 \\
\hline Bench Top Stability & LQC (15) & $14.70 \pm 0.46$ & $97.98 \pm 3.08$ & 3.14 \\
\hline \multirow{2}{*}{ (Short-term stability) } & MQC (250) & $249.33 \pm 1.29$ & $99.73 \pm 0.52$ & 0.52 \\
\hline & HQC (750) & $756.71 \pm 12.01$ & $100.89 \pm 1.62$ & 1.59 \\
\hline In-Injector Stability & LQC (15) & $14.81 \pm 0.09$ & $98.75 \pm 0.61$ & 0.61 \\
\hline \multirow{2}{*}{ (Auto-sampler stability) } & MQC (250) & $250.65 \pm 3.08$ & $100.26 \pm 1.23$ & 1.23 \\
\hline & HQC (750) & $745.68 \pm 7.11$ & $99.42 \pm 0.95$ & 0.95 \\
\hline \multirow[t]{3}{*}{ Wet extract Stability } & LQC (15) & $14.65 \pm 0.38$ & $97.63 \pm 2.56$ & 2.62 \\
\hline & MQC (250) & $250.53 \pm 8.90$ & $100.21 \pm 3.56$ & 3.56 \\
\hline & HQC (750) & $761.89 \pm 11.46$ & $101.58 \pm 1.53$ & 1.51 \\
\hline \multirow[t]{3}{*}{ Dry extract Stability } & LQC (15) & $14.88 \pm 0.37$ & $99.21 \pm 2.50$ & 2.52 \\
\hline & MQC (250) & $242.82 \pm 2.47$ & $97.13 \pm 0.99$ & 1.02 \\
\hline & HQC (750) & $746.92 \pm 7.84$ & $99.59 \pm 1.05$ & 1.05 \\
\hline
\end{tabular}

Note*: Actual concentration of AMP in ng/ml. Values are expressed in mean \pm SD, Number of samples $(n=3)$ 


\section{CONCLUSION}

The developed method possess good selectivity, specificity, there was no interference found in the plasma blanks at retention times of AMP and IS. We found a good correlation between the peak area and concentration of the drug under prescribed conditions and also the recoveries found to be $78.71 \%$ for AMP. The observation of \% RSD less than 5 for both intra-and inter-day measurements also indicates a high degree of precision. A linearity range from $5-1000 \mathrm{ng} / \mathrm{ml}$ for AMP, this linearity range covers all the strengths of AMP. The stability of AMP was found to be within the limits i.e.95.39-106.79\% concludes that there was no degradation of AMP and also stable in the plasma at different study conditions. The method found to be highly sensitivity ( $5 \mathrm{ng} / \mathrm{ml}$ ), good accuracy, precision and no matrix effect on the drug economic extraction procedure will help in further studies of AMP. Hence this method can be applied for quantifying the low levels of AMP in the biological matrix without the interference of plasma components for future investigation of AMP.

\section{ACKNOWLEDGMENT}

The author is thankful to Aurabindo pharma Ltd., Hyderabad, Telangana, India for providing drugs and to Adept Pharma and Bioscience Excellence Private Limited for providing all types of facilities for the research.

\section{AUTHORS CONTRIBUTIONS}

All the authors have contributed equally

\section{CONFLICT OF INTERESTS}

The authors report no conflict of interest

\section{REFERENCES}

1. Sharma A, Jamal MM. Opioid-induced bowel disease: a twenty-firstcentury physicians' dilemma. Considering pathophysiology and treatment strategies. Curr Gastroenterol Reports 2013;15:334.

2. FDA press release-FDA Approves Entereg to Help Restore Bowel Function Following Surgery. http://www.fda.gov/ NewsEvents/Newsroom/PressAnnouncements/2008/ucm116 899.htm. [Last accessed on 05 Jul 2018]

3. Alvimopan Product Label as approved by the FDA; 2008.

4. Neary P, Delaney P. Alvimopan. Expert Opin Invest Drugs 2005; 14:47988.
5. Schmidt WK. Alvimopan* (ADL 8-2698) is a novel peripheral opioid antagonist. Am J Surgery 2001;182(5A):27S-38S.

6. Vrushali ST, Deodhar MN, Vijayalakshmi Prakya LC. LC-MS study for simultaneous determination of tramadol hydrochloride and ketorolac tromethamine in bulk and formulation with major degradation products. Bull Faculty Pharm Cairo University 2016;54:87-97.

7. Essam Ezzeldin, Nisreen F Abo-Talib, Marwa H Tammam, Abdelaaty A Shahat. Development and validation of LC/MS/MS method for the determination of montelukast, gliclazide, and nifedipine and its application to a pharmacokinetic study. Chem Central I 2014;8:17.

8. Jaivik V Shah, Priyanka A Shah, Mallika Sanyal, Pranav S Shrivastav. Fast and sensitive LC-MS/MS method for simultaneous determination of lisinopril and hydrochlorthiazide in human plasma. J Pharm Anal 2017;7:163-9.

9. Tejas Dadhaniya, Komal Chaudhary, Priti Mehta. Development of LC-MS/MS method for determination of iloperidone in rabbit plasma: application to pharmacokinetic study. Int J Pharm Pharm Sci 2015; 7:294-7.

10. Ravi S Orugunti, H Rush A Strong, E Wells. Quantitative determination of Alvimopan in human plasma by LC-MS-MS. Worldwide Clinical Trials Drug Development SolutionsBioanalytical Sciences, Austin, Texas; T2296.

11. Addanki Padmini, Sudharani Utala, Gumparthi Prasad, Adigarla Bennayyanaidu, Koyyuri Raju, Rambabu Kuchi. A new L. C. method development and validation for analysis of atvimopin in formulations. Res Desk 2012;1:60-5.

12. US Department of health and human sciences. Food Drug Administration, Guidance for industry, Bioanalytical method development, and validation, U. S. Food Drug Administration, Center for Drug Evaluation and Research; 2001.

13. International Conference on Harmonization (ICH). Validation of Analytical Methods: Methodology. ICH Q2 B; 1996.

14. Matuszewski BK, Constanzer ML, Chavez Eng CM. Strategies for the assessment of matrix effect in quantitative bioanalytical methods based on HPLC-MS. Anal Chem 2003;75:3019-30.

15. Nicola $\mathrm{CH}$, Navgeet B, Ernest YKW. Assessing the matrix effects of hemolysed blood in bioanalysis. Bioanalysis 2009;1:105766.

16. USP (The United States pharmacopoeia convention) 30-NF 25, Rockville MD; 2007. p. 1005, 1776. 